\title{
Prominent field for shape processing and analysis of archaeological artifacts
}

\author{
Michael Kolomenkin · Ilan Shimshoni · Ayellet Tal
}

Received: date / Accepted: date

\begin{abstract}
Archaeological artifacts are an essential element of archaeological research. They provide evidence of the past and enable archaeologists to obtain qualified conclusion. Nowadays, many artifacts are scanned by 3D scanners. While convenient in many aspects, the 3D representation is often unsuitable for further analysis, due to flaws in the scanning process or defects in the original artifacts. We propose a new approach for automatic processing of scanned artifacts. It is based on the definition of a new direction field on surfaces (a normalized vector field), termed the prominent field. The prominent field is oriented with respect to the prominent feature curves of the surface. We demonstrate the applicability of the prominent field in two applications. The first is surface enhancement of archaeological artifacts, which helps enhance eroded features and remove scanning noise. The second is artificial coloring that can replace manual artifact illustration in archaeological reports.
\end{abstract}

Keywords Shape analysis $\cdot$ Shape processing $\cdot$ Archaeological models

\section{Introduction}

Man-made artifacts are a major source of our knowledge about the past. Archaeologists who study assemblages of artifacts seek to identify distinctive patterns in them, which can be used for analysis and comparison. In order to disseminate the information about artifacts, these are either illustrated in reports or scanned by 3D scanners. Nowadays, 3D representations are becoming more popular, since they provide more information by allowing the archaeologist to view the artifact from different viewpoints at various scales and perform comparative measurements. Therefore, this paper focuses on 3D representation of artifacts. Specifically, we concentrate on artifacts with reliefs, which consist of a detailed surface, the relief, that resides on the top of a smooth base surface.

M. Kolomenkin

Technion, E-mail: michkol@tx.technion.ac.il

I. Shimshoni

University of Haifa, E-mail: ishimshoni@mis.haifa.ac.il

A. Tal

Technion, E-mail: ayellet@ee.technion.ac.il 


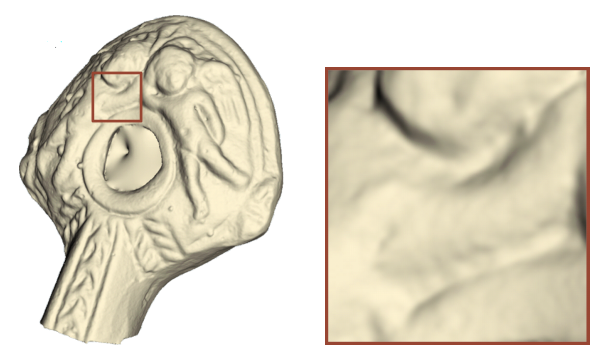

(a) Original object

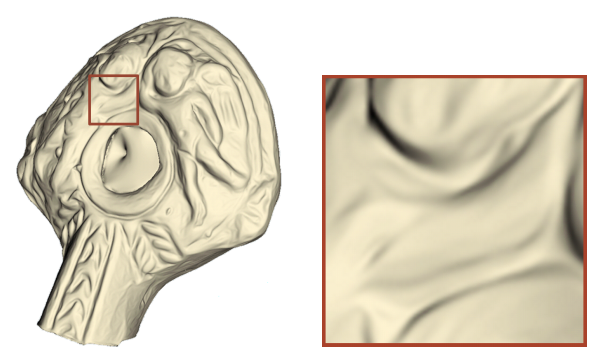

(b) Filtered object

Fig. 1 Enhancement of a late Hellenistic oil lamp from the first century BCE. The red rectangle depicts the zoomed-in part.

The main task facing the archaeologists is the analysis of these artifacts. This is done in several ways - accurately illustrating them by highlighting the surface edges, comparing artifact styles, classifying them etc. All these tasks can be facilitated by applying computer vision and computer graphics techniques [2,13,20,29]. However, the $3 \mathrm{D}$ representation is often flawed, either due to defects in the original artifacts or due to the erosion the artifact underwent after spending two thousand years underground. Noise added in the scanning process may also damage the representation. Figure 1(a) gives an example of a flaw. The surface of the original object is slightly rippled and looks blurred.

This paper addresses this problem by proposing a novel framework for processing the artifacts. It is based on a definition of a new direction field (a normalized vector field), termed the prominent field, defined for every point on the surface. This field is constructed in a manner in which it is smooth on the surface. Intuitively, the direction of this prominent field, termed the prominent direction, is perpendicular to the surface's feature curves. With respect to the reliefs, we show that the prominent field is superior to previously proposed vector fields. The existing fields are oriented mostly according to the principal directions, which do not always coincide with the feature curves of the surface.

Since the prominent field is closely related to the feature curves of the relief surface, it is beneficial for a variety of processing applications. We demonstrate its effectiveness in two applications: surface enhancement and artificial coloring. The goal of adaptive filtering is to enhance the features while keeping the surface intact. This may also help to remove the scanning noise. We propose to smooth the surface using the prominent field along the feature curves and enhance it in the prominent direction. Figure 1(b) shows the effect of the adaptive filtering. The filtered object is smoother and has crisper, more visible details than the original object.

As a second application, we present a method for artificially coloring objects. The key idea is to color the surface according to its normal curvature in the prominent direction. This coloring increases the color contrast on the feature curves, thus enhancing them.

The contribution of this paper is hence threefold:

- We define the prominent field and show how to compute it in interactive time.

- We show how to employ the prominent field for surface smoothing and enhancement.

- We propose a method for artificial surface coloring that emphasizes the object features.

The paper continues as follows. Section 2 presents the required background. Section 3 defines the prominent field and shows how to compute it. Sections 4 and 5 demonstrate the applications. Finally, Section 6 concludes the paper. A preliminary version of this work appeared in [16]. 


\section{Background}

This section presents the essential background on relief surfaces and on vector fields on surfaces.

Relief surfaces and feature curves on them: A relief surface can be viewed locally as a terrain. Like any other terrain, it has valleys and ridges. Three types of feature curves are defined on it: ridges, valleys, and relief edges, as illustrated in Figure 2(a). All these curves run on the slopes of the terrain, which can be approximated locally by the step edge model (Figure 2(b)).

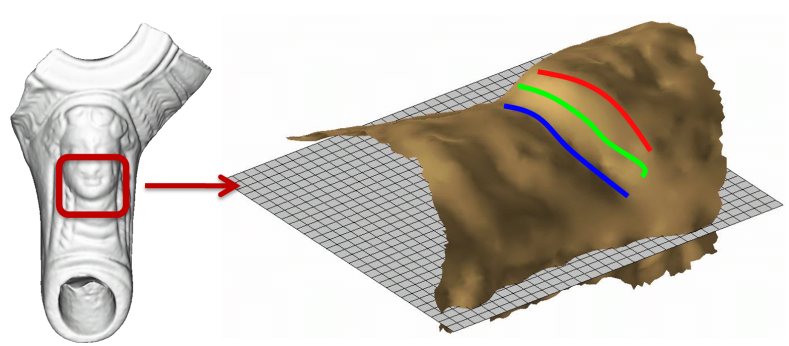

(a) Local terrain

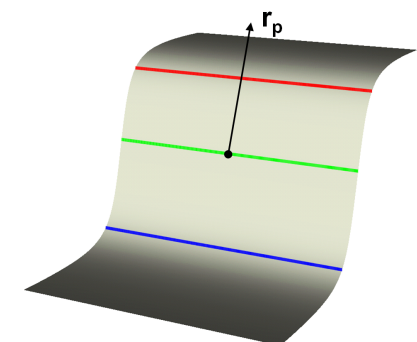

(b) Step edge model

Fig. 2 Three types of feature curves on a relief surface. (a) The Hellenistic oil lamp can be viewed locally as terrain. The terrain has ridges (red), valleys (blue), and relief edges (green). (b) The step edge model can approximate the slopes of the terrain.

Ridges and valleys are similar to their geographical counterparts and usually indicate sharp changes in the surface orientation. Ridges (valleys) are defined as the maximum (minimum) of the normal curvature in the first principal direction [5].

On ideal step edges, relief edges run on the slopes between ridges and valleys and are parallel to them. They are shown to correspond to the image edges of the local image $I$ [15]. Equivalently, they are defined as zero crossings of the curvature in the direction of the step edge model that best approximates the surface locally in the $L_{2}$ norm. The direction is termed the edge direction.

Figure 3 compares different types of curves. Ridges are erroneous for this model; valleys make the dancers wider and do not always follow precisely the figures outline; relief edges provide more accurate results.

Vector fields on surfaces: A vector field is a vector asociated with every point on the surface. Vector fields on surfaces are essential for many graphics applications, such as texture synthesis [30,28], non photo-realistic rendering [11], fluid simulation [22], shape deformation [9] and others. There exists a variety of papers on editing, generation, manipulation, and filtering of vector fields [32,7]. Since the magnitude of the vector field is irrelevant for our work, we will restrict the discussion to direction fields.

The most common candidates for direction fields are the principal directions, which correspond to the directions of the maximal and the minimal curvatures. The principal directions are reliable near ridges and valleys, i.e. at locations where the ratio of the principal curvatures is high, but are ill-defined near umbilical points. Therefore, typically the field is computed so as to coincide with the reliable principal directions and be smooth at other points $([11,19])$. While the principal directions are useful for general objects, they are less 


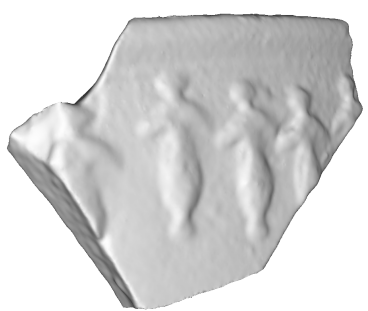

(a) Original object

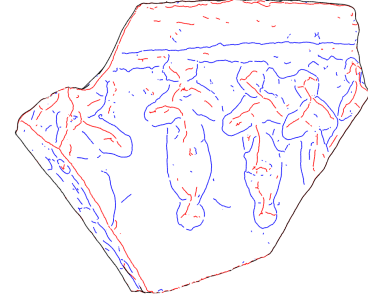

(b) Ridges and valleys

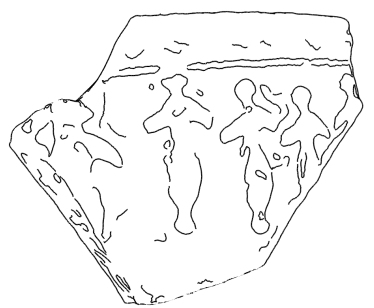

(c) Relief edges

Fig. 3 Three types of feature curves on a Hellenistic vase depicting five dancers. Ridges (red) \& valleys (blue) do not follow precisely the dancers, whereas the relief edges (black) are more accurate.

suitable for objects with reliefs. This is so since the relief details are often close to umbilical and the principal directions might fail to capture their orientation correctly. Figure 4(b) shows that while the principal directions are oriented well near the ridges and the valleys, they are noisy at other places.

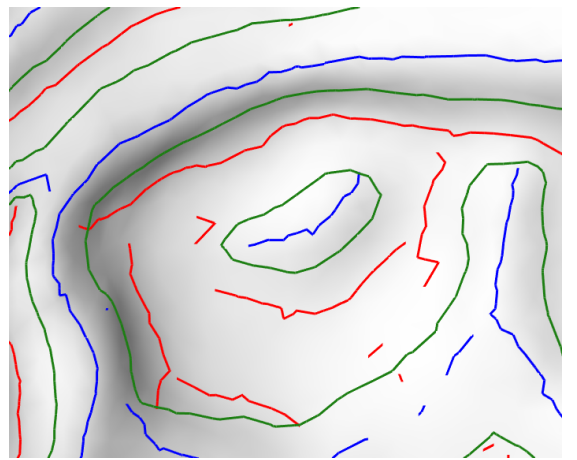

(a) Original object

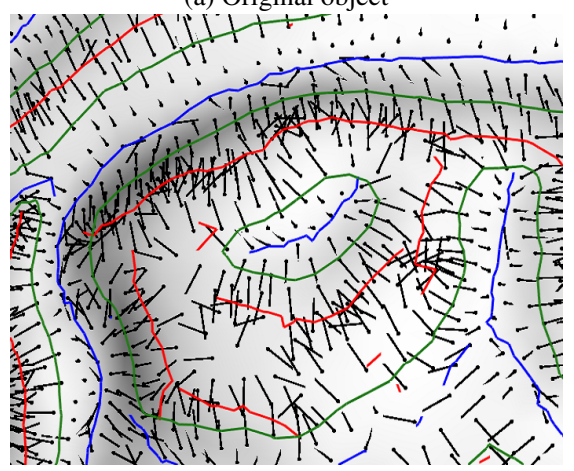

(c) Relief direction field

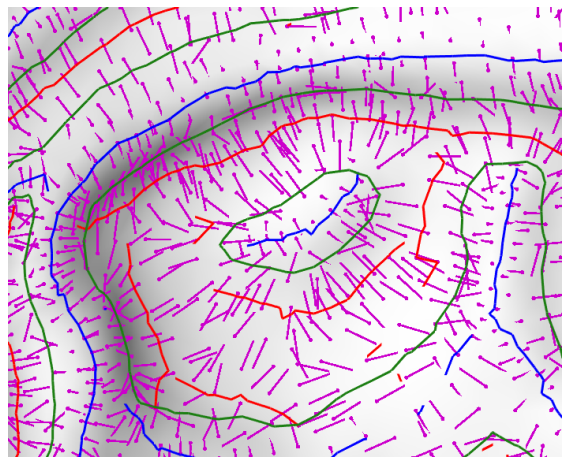

(b) Principal direction field

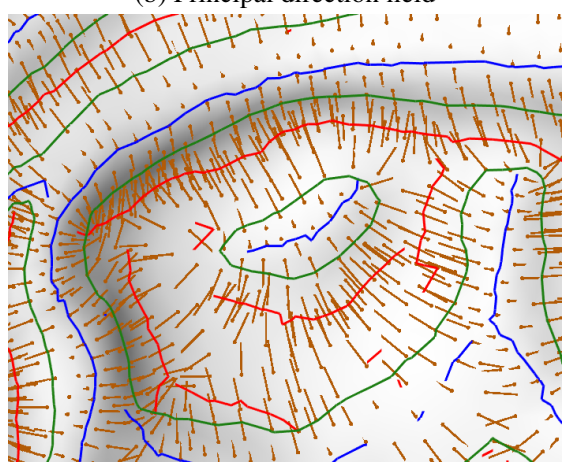

(d) Prominent direction field

Fig. 4 Different direction fields. In (a) the ridges (red), valleys (blue), and relief edges (green) are depicted. The principal direction field (magenta) is oriented well near the ridges and the valleys, but noisy at other places (b). The relief direction (black) is oriented well on the relief edges, but not at other parts of the surface (c). Our prominent direction (orange) is oriented well everywhere on the surface. 
The directions perpendicular to demarcating curves [14] and to relief edges [15] are more appropriate for relief surfaces, since these curves are designed to illustrate relief features. The direction perpendicular to demarcating curves is the direction of the curvature gradient (the direction of the maximal curvature derivative). The direction perpendicular to relief edges is the direction of the locally best-fitting step edge. These directions are well defined on the relief edges, but are meaningless at the other parts of the surface, and thus cannot be used in a straightforward manner. Figure 4(c) shows that the relief directions are well oriented near the relief edges, but are randomly oriented at other locations.

\section{Prominent field}

This section presents a novel direction field - the prominent field. Intuitively, the prominent field is defined as the smoothest field perpendicular to the object features. A field satisfying this definition can enable us for example to enhance features in the direction of the field, while removing noise in the perpendicular direction.

Below we first present the surface model. Next we define the prominent field, first on the feature curves and then on the whole surface. Finally, we describe the algorithm for computing the prominent field on polygonal meshes.

Surface model: We define a relief surface as a surface composed of a smooth, low-frequency base and a high-frequency height function [15]. This function represents the signed distance between the base to the surface in the direction of the base's unit normal. (See Figure 5.) This decoupling of the surface into the base and the height function is unknown.

Formally, given a surface $S(u, v): \mathbb{R}^{2} \rightarrow \mathbb{R}^{3}$, we assume that it consists of a smooth base $B(u, v): \mathbb{R}^{2} \rightarrow \mathbb{R}^{3}$ and a height function $I(u, v): \mathbb{R}^{2} \rightarrow \mathbb{R}$ defined on $B:$

$$
S(u, v)=B(u, v)+\overline{\mathbf{n}}(u, v) I(u, v)
$$

where $u$ and $v$ are the coordinates of a parameterization and $\overline{\mathbf{n}}(u, v): \mathbb{R}^{2} \rightarrow \mathbb{S}^{2}$ is the normal of $B\left(\mathbb{S}^{2}\right.$ being the unit sphere). We assume that $B$ is locally a manifold and that its curvature has a smaller value than the curvature of $I$.

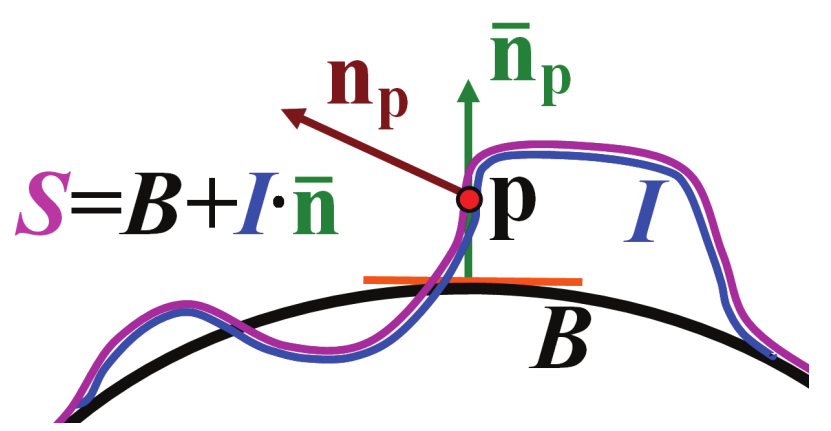

Fig. 5 The surface $S$ (magenta) is composed of a smooth base $B$ (black) and a function $I$ (blue). Function $I$ at point $\mathbf{p}$ can be locally viewed as an image defined on the tangent plane (orange) of the base. Point $\mathbf{p}$ is a relief edge point if it is an edge point of this image. The normal $\mathbf{n}_{\mathbf{p}}$ (brown) is the normal of $S$ and $\overline{\mathbf{n}}_{\mathbf{p}}$ (green) is the normal of $B$ corresponding to $\mathbf{p}$. 
The prominent field on features: We are seeking a smooth direction field - the prominent field - that is perpendicular to the object feature curves. This direction is important since many processing tasks are closely related to the feature direction (which should be maintained, enhanced, etc.). Smoothness is required in order to produce smooth results.

Had the surface be an ideal step edge, this direction would be perpendicular to the ridge, valley, and relief edge (Figure 2(b)). In practice however, surfaces are not ideal step edges (Figure 2(a)) and thus we need to define a direction field more carefully.

To address this problem, we divide the surface points near the feature curves into two fuzzy classes. The first class consists of the points residing near the ridges and the valleys. Here the prominent direction should be equal to the first (maximum) principal direction. The second class includes points residing near the relief edges. In this case the prominent direction should be equal to the relief direction. The classification is fuzzy and hence the regions may overlap.

Formally, let $\mathbf{p}$ be a point on the surface, $\mathbf{g}_{\mathbf{p}}$ be the relief direction, and $\mathbf{t}_{\mathbf{p}}$ be the first principal direction. As mentioned above, on the step edge model, $\mathbf{g}_{\mathbf{p}}$ is meaningful only on the relief edge and $\mathbf{t}_{\mathbf{p}}$ is well-defined near ridges and valleys. Therefore, to define the prominent direction $\mathbf{r}_{\mathbf{p}}, \mathbf{g}_{\mathbf{p}}$ and $\mathbf{t}_{\mathbf{p}}$ are combined as a weighted combination, where the weights are proportional to the likelihood of the point to be near a relief edge. Therefore, the weight $\alpha_{\mathbf{p}}$ is 1 at relief edges and 0 at ridge/valley points.

Definition 31 The prominent direction is

$$
\mathbf{r}_{\mathbf{p}}=\alpha_{\mathbf{p}} \mathbf{g}_{\mathbf{p}}+\left(1-\alpha_{\mathbf{p}}\right) \mathbf{t}_{\mathbf{p}}
$$

where $\alpha_{\mathbf{p}} \in[0,1]$ is a scalar weight that determines the relative distance of $\mathbf{p}$ from the relief edge.

The question is how to define $\alpha_{\mathbf{p}}$. Let $\kappa_{1}$ and $\kappa_{2}$ be the principal curvatures, $k$ their ratio, and $l$ the median length of mesh edges. Empirically, we observed that good results are obtained when

$$
\alpha_{\mathbf{p}}=\left\{\begin{array}{cc}
1 & \max \left(\left|\kappa_{1}\right|,\left|\kappa_{2}\right|\right)<3 / l \text { or }|k|<2 \\
0 & |k|>4 \\
\frac{4-|k|}{2} & \text { otherwise. }
\end{array}\right.
$$

The intuition behind this definition is as follows. On a relief edge $\left(\alpha_{\mathbf{p}}=1\right)$, the principal curvatures should be small (on an ideal step edge, the edge point is planar) with respect to the surface resolution (thus, the use of $l$ ). If the relief edge bends, the ratio of the principal curvatures is small. On a valley or a ridge $\left(\alpha_{\mathbf{p}}=0\right)$, the ratio between the principal curvatures is large.

The prominent direction on the whole surface: In the previous section we defined the prominent field on the feature curves. To extend the definition to the whole surface, we search for the smoothest direction field that satisfies the values of the prominent field on the features.

Utilizing the Laplacian as the smoothness measure, we define the prominent field as the solution of the Poisson equation. The values of the prominent field on the features serve as boundary conditions for the equation.

Formally, we want to compute the prominent field $\mathbf{s}_{\mathbf{p}}=\left[s_{\mathbf{p}}^{u}, s_{\mathbf{p}}^{v}\right]$, such that the Laplacian $\left[\Delta s_{\mathbf{p}}^{u}, \Delta s_{\mathbf{p}}^{v}\right]$ of the field components is equal to zero and $\mathbf{s}_{\mathbf{p}}=\mathbf{r}_{\mathbf{p}}$ on the features. Let $\beta_{\mathbf{p}} \in[0,1]$ be our confidence that $\mathbf{p}$ is a feature point (explained below). At each point $\mathbf{p}$, the following 
should hold:

$$
\begin{aligned}
\beta_{\mathbf{p}} \mathbf{s}_{\mathbf{p}} & =\beta_{\mathbf{p}} \mathbf{r}_{\mathbf{p}}, \\
\left(1-\beta_{\mathbf{p}}\right) \Delta s_{\mathbf{p}}^{u} & =0 \\
\left(1-\beta_{\mathbf{p}}\right) \Delta s_{\mathbf{p}}^{v} & =0 .
\end{aligned}
$$

Thus, on the features $\left(\beta_{\mathbf{p}} \approx 1\right)$, the first equation enforces the boundary conditions and elsewhere, the two other equations enforce the smoothness of the solution.

We approximate the confidence value $\beta_{\mathbf{p}}$ such that it is close to one when the point is near an edge and zero otherwise. Recall that the points on the edge are characterized either by a high ratio between the principal curvatures (near ridges and valleys) or by a small difference between the surface $S$ and its approximated step edge $\varepsilon_{r}$ [15, Equation 7]. Specifically,

$$
\beta_{\mathbf{p}}=\left\{\begin{array}{l}
1\left(|k|>2 \text { and } \max \left(\left|\kappa_{1}\right|,\left|\kappa_{2}\right|\right)>3 / l\right) \text { or } \varepsilon_{r}<\theta / l \\
0 \quad \text { otherwise }
\end{array}\right.
$$

where $\theta$ is a user controlled threshold. The threshold enables the user to determine what points are considered edges and directly influence the appearance of the prominent field. We observed that setting $\theta$ to 2 gives good results in most cases.

Computation of the prominent field: In practice, our surface is given as a triangular mesh. We need to compute $\left[s_{\mathbf{p}}^{u}, s_{\mathbf{p}}^{v}\right]$ for every vertex of the mesh, and the entire collection will constitute a field. Let $\mathbf{p}$ be a vertex of the mesh and $N(\mathbf{p})$ be the set of the neighbors of $\mathbf{p}$ (i.e., vertices that share a mesh edge with $\mathbf{p}$ ).

To compute the prominent field $\mathbf{s}_{\mathbf{p}}$ we need to solve Equation 3. In order to perform this efficiently, we restrict ourselves to solving a couple of systems of linear equations. We do it by first deriving a linear approximation of the Laplacian $\left[\Delta s_{\mathbf{p}}^{u}, \Delta s_{\mathbf{p}}^{v}\right]$ of the components of the prominent field and then solving the set of linear equations in $\mathbf{s}_{\mathbf{p}}$.

To compute the Laplacian of a scalar function $f$ on a mesh, we follow [17]:

$$
\begin{aligned}
\Delta f(\mathbf{p}) & =\frac{1}{2 A} \sum_{j \in N(\mathbf{p})}\left(\cot \left(\gamma_{j}\right)+\cot \left(\delta_{j}\right)\right)\left(f(\mathbf{p})-f\left(\mathbf{p}_{j}\right)\right) \\
& \equiv \frac{1}{2 A} \sum_{j \in N(\mathbf{p})} w_{j}\left(f(\mathbf{p})-f\left(\mathbf{p}_{j}\right)\right), w_{j} \equiv \cot \left(\gamma_{j}\right)+\cot \left(\delta_{j}\right),
\end{aligned}
$$

where $A$ is the area of the Voronoi cell of $\mathbf{p}$, and $\gamma_{j}$ and $\delta_{j}$ are the angles opposite the edge $\left[\mathbf{p}, \mathbf{p}_{j}\right]$ of the triangles sharing this edge.

$\left[\Delta s_{\mathbf{p}}^{u}, \Delta s_{\mathbf{p}}^{v}\right]$ cannot be computed directly using Equation 5, since the components $\left[s_{\mathbf{p}}^{u}, s_{\mathbf{p}}^{v}\right]$ of the prominent field are not scalar functions of the surface. Rather, they are defined in the local tangent plane, which differs from point to point. To address this problem, we calculate the transformation between the local coordinate systems of the neighboring vertices and utilize it in the computation of the Laplacian.

Given a vertex $\mathbf{p}$ and its neighbor $\mathbf{p}_{j}$, this transformation is computed as follows. First, the tangent plane of point $\mathbf{p}_{j}$ is rotated by aligning the normals of $\mathbf{p}$ and $\mathbf{p}_{j}$. Next, the coordinates systems are aligned in the tangent plane, by applying a $2 \mathrm{D}$ rotation by $\theta: R=$ $\left[(\cos \theta, \sin \theta)^{T},(-\sin \theta, \cos \theta)^{T}\right]$, where $\theta$ is the angel between the rotated first principal 
directions (Figure 6). Finally, the Laplacian of the prominent field can be written as:

$$
\begin{aligned}
& \Delta s_{\mathbf{p}}^{u}=\frac{1}{2 A} \sum_{j=N(\mathbf{p})} w_{j}\left(s_{\mathbf{p}}^{u}-\cos \theta s_{\mathbf{p}}^{u}-\sin \theta s_{\mathbf{p}}^{v}\right), \\
& \Delta s_{\mathbf{p}}^{v}=\frac{1}{2 A} \sum_{j=N(\mathbf{p})} w_{j}\left(s_{\mathbf{p}}^{v}+\sin \theta s_{\mathbf{p}}^{u}-\cos \theta s_{\mathbf{p}}^{v}\right) .
\end{aligned}
$$

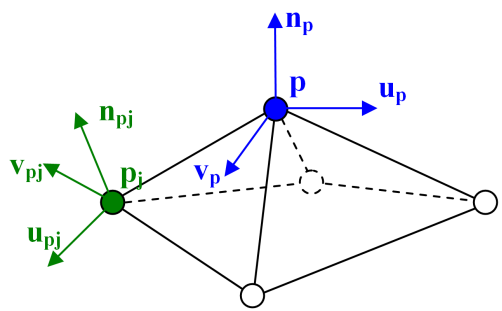

(a) Initial positions

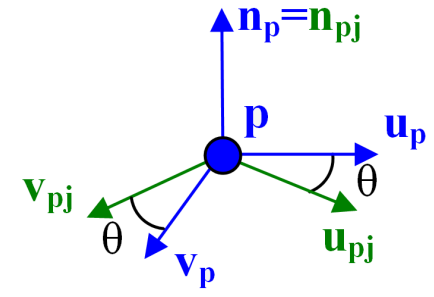

(b) After aligning the normals

Fig. 6 Alignment of the local coordinate systems. (a) First, we rotate the coordinate system of $\mathbf{p}_{j}$ so that the tangent plane of $\mathbf{p}_{j}$ coincides with the tangent plane of $\mathbf{p}$. The rotation is performed around the cross product of $\mathbf{n}_{\mathbf{p}}$ and $\mathbf{n}_{\mathbf{p}_{j}}$. (b) Then, the coordinate systems of $\mathbf{p}_{j}$ and $\mathbf{p}$ are registered by rotating the rotated tangent plane of $\mathbf{p}_{j}$ by $\theta$.

Finally, substituting equations 4 and 6 into Equation 3 yields a system of linear equations, whose unknowns are the components of the prominent field $\mathbf{s}_{\mathbf{p}}$. This is solved using a standard sparse linear equation solver. Since the prominent field is a direction field, it is then normalized. Note that after normalization, the Laplacian is not guaranteed to remain small. In practice, however, the change is negligible.

Figure 7 compares the prominent field to the other direction fields. It can be seen that the principal direction field and the relief direction field are inappropriate in most surface locations, whereas our prominent field is both in the desired direction near the feature curves and smooth everywhere.

\section{Application: Surface enhancement and smoothing}

Scanned archaeological objects are often unsuitable for further processing and visual analysis, either due to erosion that they underwent during the ages or due to scanning noise. This section describes how our prominent field can be utilized to enhance and smooth these objects, to enable effective processing and analysis.

One way to address these problems is by using adaptive filtering algorithms, which smooth (or denoise) the surface, while keeping the features intact or enhancing them. Existing approaches for adaptive filtering on meshes operate either on the mesh vertices [4, $8,31]$, the mesh normals $[18,24]$, or the curvatures [6]. The techniques differ in the energy functional they attempt to minimize.

While these approaches perform well preserving and enhancing ridges and valleys, they are not designed for relief objects. In particular, there are a couple of cases in which they may produce inferior results. The first case occurs when no distinct ridges or valleys can 


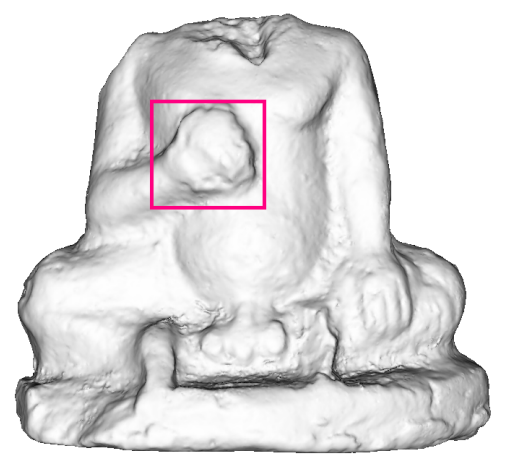

(a) Original object

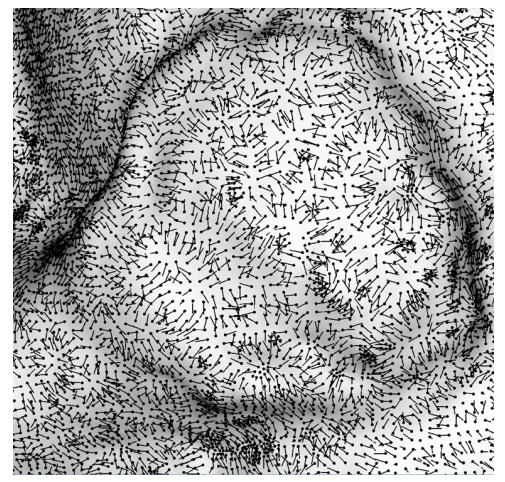

(c) Relief field

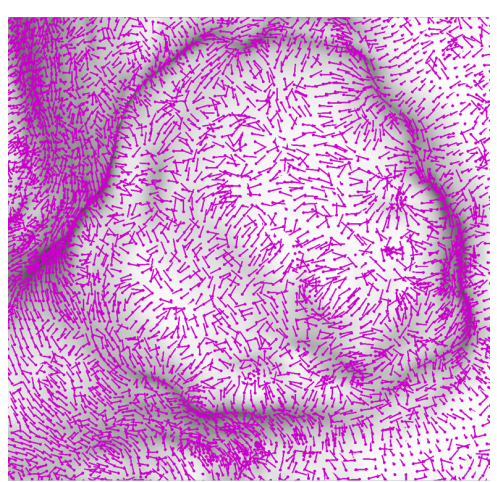

(b) Principal field

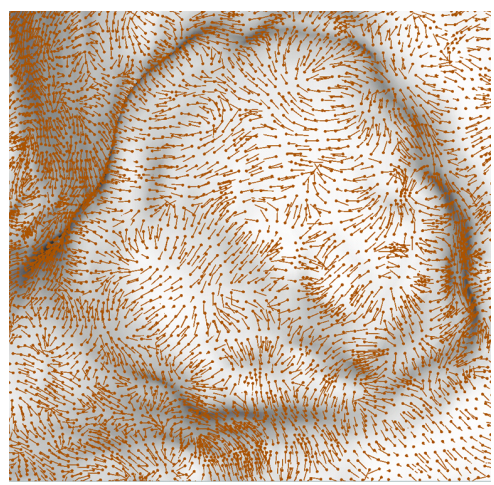

(d) Prominent field

Fig. 7 Direction fields. The principal directions (b) and the relief directions (c) lack meaning far from their respective feature curves, in contrast to the prominent field, which is in the desired direction near the feature curves and smooth everywhere (d).

be detected on the surface. These approaches will simply smooth the objects, diminishing the $3 \mathrm{D}$ features. The second case occurs when there exist distinct valleys and ridges, but the slope of their step edge is shallow, as illustrated in Figure 8. In this case, these approaches aim at enhancing each of these features separately, but do not enhance the step edge model between them. Our goal is to preserve and enhance this step edge by steepening the slope of the step edge.

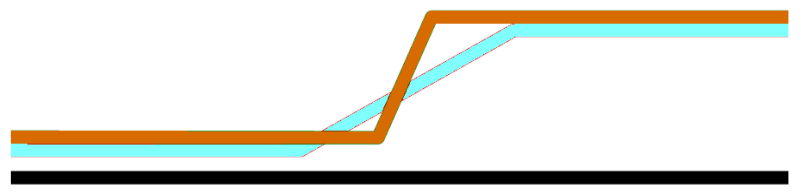

Fig. 8 The cyan curve is the local image defined on the black base. Since this surface has sharp ridges and valleys, it will not be enhanced by standard adaptive filtering. The desired result, illustrated in orange, enhances the 3D feature. 
We propose a novel approach that solves these problems. It consists of two steps bilateral filtering and inverse curvature flow - each makes use of our prominent field to guide the smoothing and enhancement directions. Though we describe a specific bilateral filtering, our prominent field can be combined with many other adaptive filtering techniques.

Step 1 - Bilateral filtering: A bilateral filter sets the position of a vertex to a weighted average of its neighbors. The weights depend both on the distance between the vertices and on their similarity. We propose to base the similarity component on the distance between the vertices along the prominent direction.

Let $\mathbf{p}$ be a vertex on the mesh, $N(\mathbf{p})$ be the set of its neighbors, $d_{j}=\left\|\mathbf{p}-\mathbf{p}_{j}\right\|$ be the Euclidean distance between $\mathbf{p}$ and one of its neighbors $\mathbf{p}_{j}$, and $\mathbf{n}_{\mathbf{p}}$ be the normal at $\mathbf{p}$. In [8] it is proposed to define the similarity as the distance between $\mathbf{p}_{j}$ and $\mathbf{p}$ 's tangent plane: $h_{j}=\left|\left\langle\mathbf{n}_{\mathbf{p}}, \mathbf{p}-\mathbf{p}_{j}\right\rangle\right|$, so that smoothing is performed when $\mathbf{p}_{j}$ is close to the tangent plane of $\mathbf{p}$. We propose to add to this definition a term that depends on $r_{j}$, the projection of $\mathbf{p}-\mathbf{p}_{j}$ along the prominent direction. Thus, smoothing will not be performed in the prominent direction, which prevents $3 \mathrm{D}$ feature blurring. This is done by multiplying the weights suggested in [8] by the term $e^{-r_{j}^{2} / 2 \sigma_{p}^{2}}$.

Hence, our similarity-based change of $\mathbf{p}$ in its normal direction is

$$
\delta_{\mathbf{p}}=C \sum_{j \in N(\mathbf{p})} e^{-d_{j}^{2} / 2 \sigma_{c}^{2}} \cdot e^{-h_{j}^{2} / 2 \sigma_{s}^{2}} \cdot e^{-r_{j}^{2} / 2 \sigma_{p}^{2}} \cdot h_{j},
$$

yielding a new position for $\mathbf{p}$ :

$$
\mathbf{p}^{\prime}=\mathbf{p}+\delta_{\mathbf{p}} \mathbf{n}_{\mathbf{p}}
$$

where, $C$ is the normalization coefficient. In the implementation, $\sigma_{s}=0.5 \sigma_{c}, \sigma_{s}=0.4 \sigma_{c}$, and $\sigma_{c}$ is a user-supplied parameter that determines the amount of smoothing. It is common to slightly smooth the object prior to computing the distances.

Figures 9(c) \& 10(c) show the results obtained by applying our bilateral filtering to scans of real archaeological artifacts. In comparison to [8] (Figures 9(b) \& 10(b)) it can be seen that the features are more pronounced.

Step 2 - Inverse-curvature flow: The inverse-curvature flow is a high frequency filter [1, $25,26]$, which updates the position of a vertex so as to increase the absolute value of its curvature. It can be based on the mean, maximum, minimum, or any other type of curvature.

While the inverse-curvature flow manages to enhance features, it suffers from two drawbacks. First, it often creates spurious features on the surface, in addition to the enhanced features. This is so since in near-flat region points with locally higher-curvature values are enhanced. Second, it is an iterative process that does not have a well-defined stopping criterion, causing unnaturally exaggerated features.

We propose a new inverse-curvature flow, which is based on two modifications to the standard flow. To solve the first problem, the curvature is computed in the prominent direction, enhancing only the real features. To solve the second problem, a new stopping criterion is suggested, which is based on the intuition in which a point should not exceed the extremum of the height function in the neighborhood of the point. Figure 11 illustrates the problem and our solution. It can be seen that the standard inverse curvature flow results in an exaggerated edge (magenta) that exceeds the original height in the neighborhood of the point (cyan). Our edge (orange) stops when it reaches the maximal height, resulting in a more appealing surface.

To perform this computation, we need to estimate the relative height function over the base. However, the decoupling of the surface into a base and a height function is unknown 


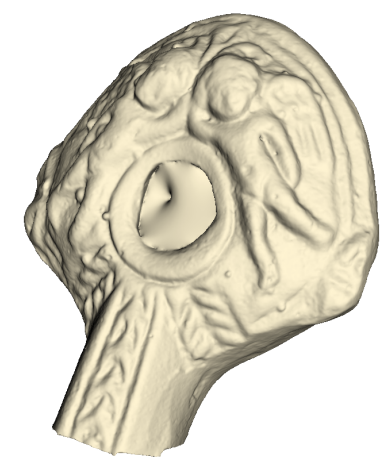

(a) The given object

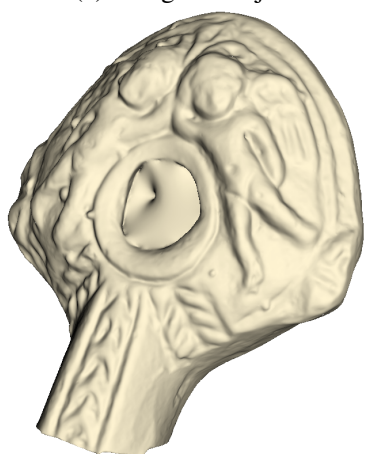

(c) The result of our bilateral filtering (Step 1)

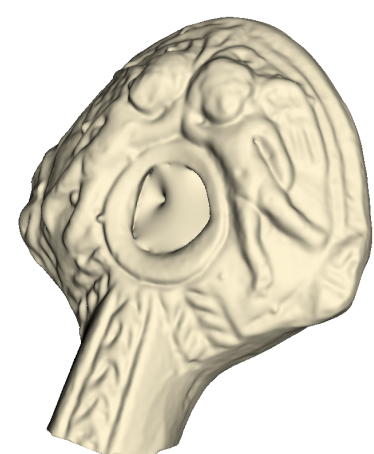

(b) The result of the bilateral filtering of [8]

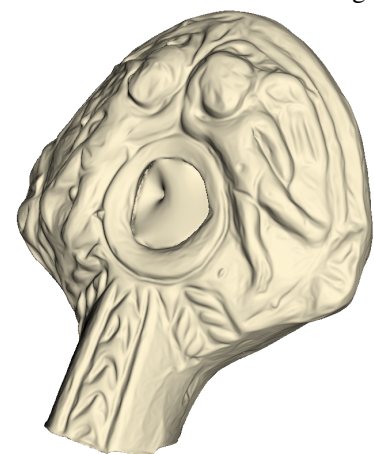

(d) Our final result

Fig. 9 Enhancement of a late Hellenistic oil lamp from the first century BCE. In our result, the limbs of the cupid, as well as the ornaments, are more pronounced.

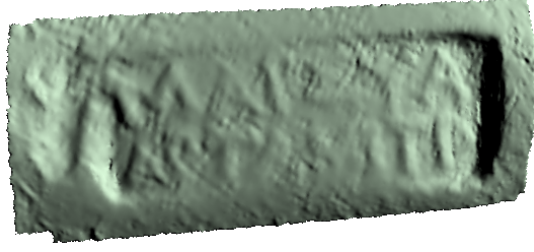

(a) The given object

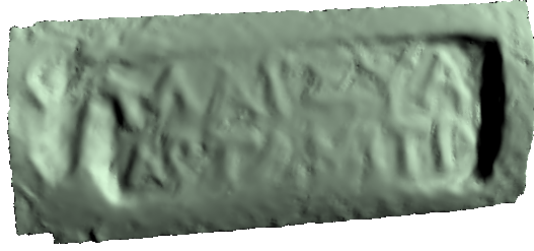

(c) The result of our bilateral filtering (Step 1)

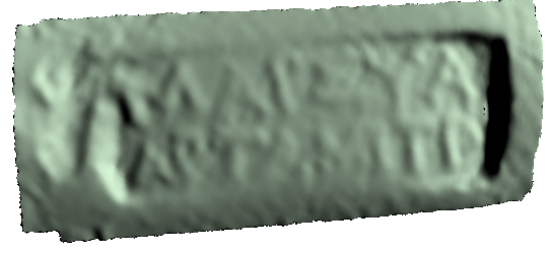

(b) The result of the bilateral filtering of [8]

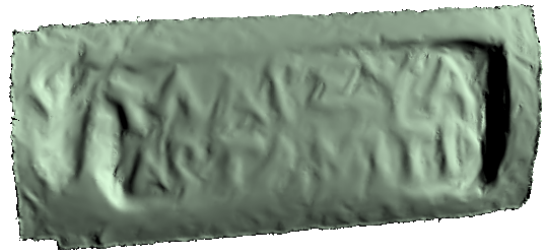

(d) Our final result

Fig. 10 Enhancement of a Hellenistic handle stamped by a Greek official. In our result, the letters are crisper, whereas the bumpy background is smoothed. 


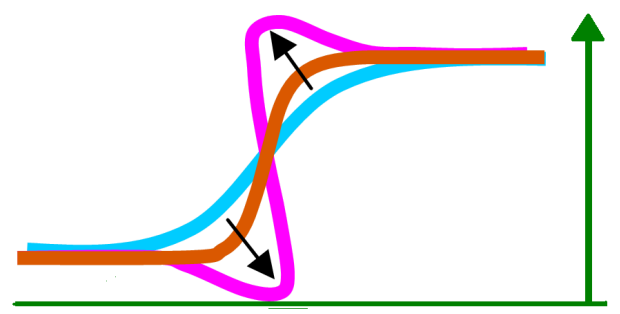

Fig. 11 Inverse-curvature flow. The initial surface is in cyan; the standard inverse-curvature flow is in magenta, and our inverse-curvature flow is in orange. The base is the green line and the normal to the base is the green arrow. Our inverse-curvature flow does not exceed the maximum (minimum) local height.

(Figure 5). To approximate this decoupling, it suffices to estimate the normal to the base at each point [15]. To estimate the base normals, the surface $(S)$ normals are smoothed at the neighborhood of the point. Our approach utilizes an adaptive Gaussian filter, similarly to $[18,15]$.

Results: Figures 9-10 illustrate some results and comparisons. Figure 9 shows a Hellenistic oil lamp. The original object is slightly eroded and has small ripples on the surface. Standard bilateral filtering succeeds to remove the ripples but the details become blurred. Our bilateral filtering (Step 1) causes the blurring (e.g., on the legs), but the result can still be enhanced. After Step 2 of our algorithm, the surface becomes smooth while the details become crisper. For instance, the torso of the cupid on the right is smooth while his arms, legs, and wings are more clearly visible. Figure 10 depicts a Hellenistic handle stamped by a Greek official. The defects on the original scan are removed by both bilateral filters, but the letters in our final result are more recognizable and protruding.

Additional results are shown in Figures 12-14. After applying our algorithm, the face in Figure 12 has much clearer facial features. Note especially the quality of the nose, the eyes, and the crown. Figure 13 displays an Ottoman pipe. Our algorithm keeps intact even the smallest carvings on the pipe. Figure 14 displays a shard of a Hellenistic vase. In this example the user decided to stop the iterations of the algorithm before the automatic stopping criterion was reached. The resulting object (Figure 14(a)) looks more appealing and has a crisper ornament. Zooming in to the details of the shard (Figure 14(b)) it can be seen that the quality of our result is even more visible. For instance, the S-shaped ornament is smoother and has clearer boundaries.

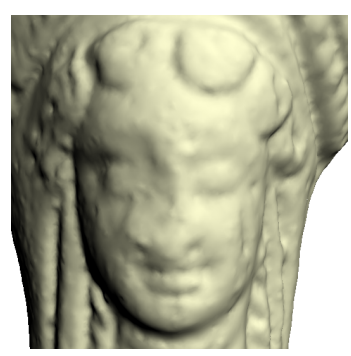

(a) The given object

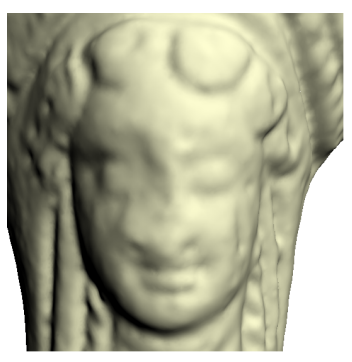

(b) After our bilateral filtering

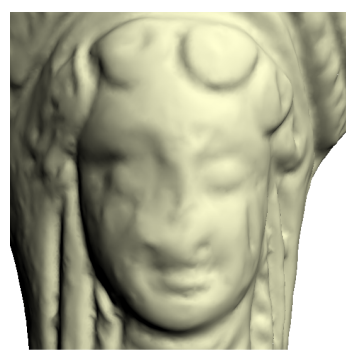

(c) Our final result

Fig. 12 A late Hellenistic oil lamp from the first century BCE. Note especially the eye on the left from which the noise has been removed and its shape is more pronounced. 


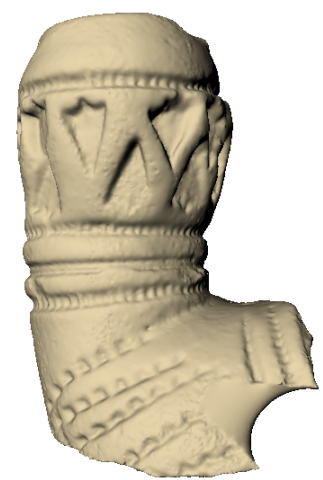

(a) The given object

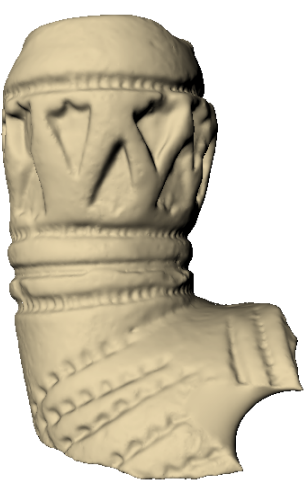

(b) After our bilateral filtering

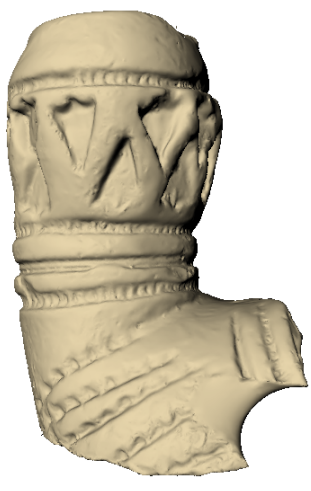

(c) Our final result

Fig. 13 An Ottoman pipe. Note the quality of small carvings which were enhanced by the algorithm.

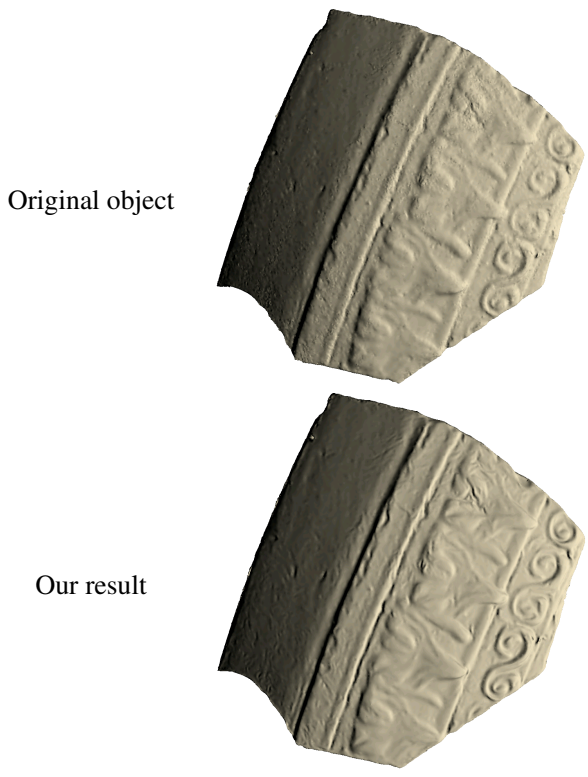

(a) The full object
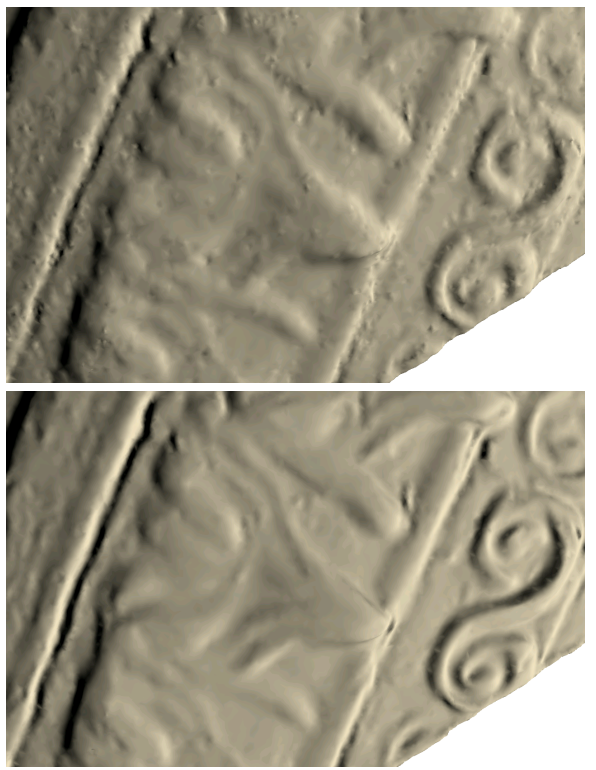

(b) Zoom in

Fig. 14 A shard of a Hellenistic vase. The algorithm removes the noise visible on the left while enhancing the $\mathrm{S}$ shaped decoration on the right.

\section{Application: Prominent coloring}

Traditionally, archaeological artifacts are drawn by hand and printed in the reports of archaeological excavations, as illustrated in Figure 15. The artists utilize artificial coloring in order to enhance the three-dimensional features. Several kinds of computerized artificial 
coloring methods have been proposed in the literature [3,10,12,21,27], in which the object is colored according to its geometric properties. For instance, it is proposed in [12] to color each point on the surface according to its mean or maximal curvature.

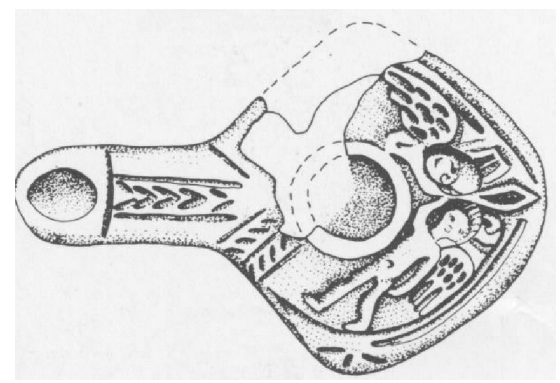

Fig. 15 Manual illustration of an archaeological artifact [23]

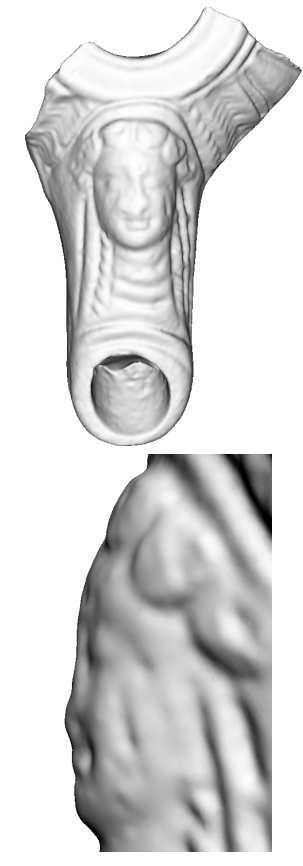

(a) The object
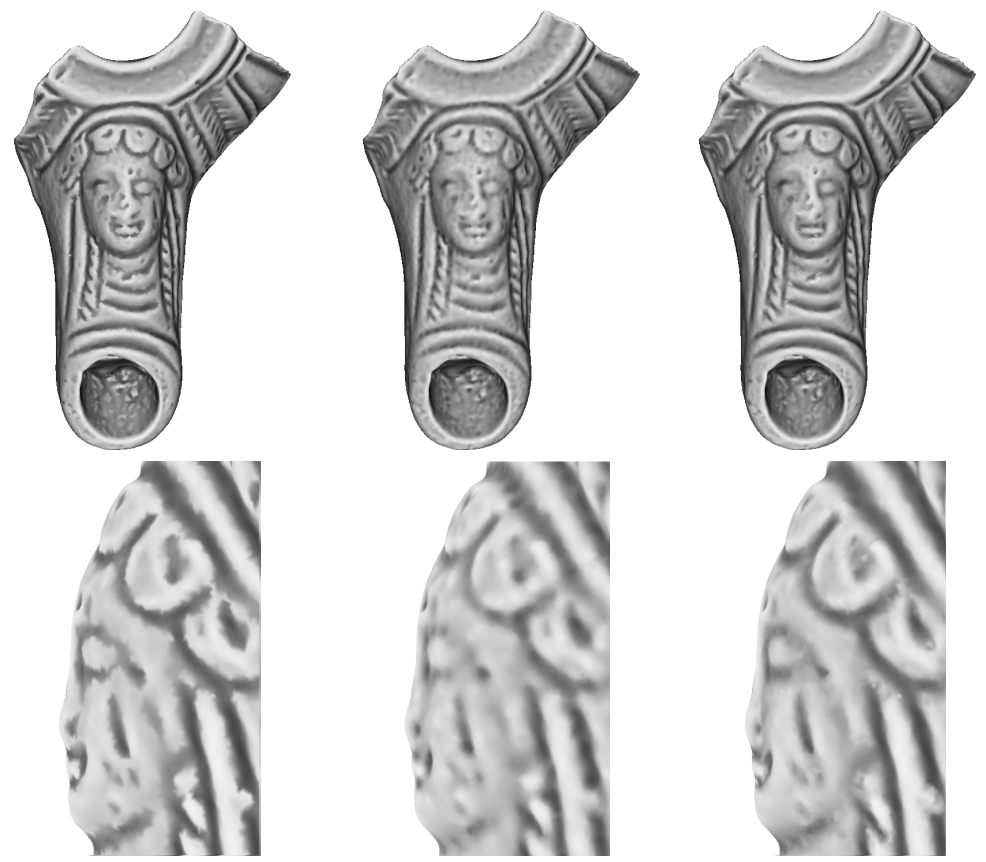

(b) Max-curvature coloring

(c) Mean-curvature coloring

(d) Prominent coloring

Fig. 16 Comparison of various coloring methods. Top: complete artifact; bottom: partial profile. Note that the maximal-curvature coloring is noisy; the mean-curvature coloring is blurred; our coloring is crisper and less noisy. This is visible, for instance, on the eye, crown, and hair.

We propose a new method for artificial coloring, termed prominent coloring. The color of a vertex is set according to its curvature in the prominent direction. The lower the curvature, the darker its color. Formally, given a vertex with prominent curvature $\kappa_{p}$, its color is 
defined as

$$
\text { color }=\arctan \left(\lambda \kappa_{p}\right),
$$

where $\lambda$ is a user supplied parameter controlling the overall image contrast.

Results: Figures 16-18 illustrate our coloring method and compares it to mean and maximal curvature colorings. Prominent coloring can emphasize poorly visible features. For instance, the scar on the cheek and the cavity on the crown of the person in Figure 16 are not clearly seen on the original scan. With our prominent coloring, they are easily detectable.

In general, the maximal curvature coloring is noisy and jagged since the maximal curvature is sensitive to noise and to small surface variations. The mean curvature coloring is blurred since it depends also on the base surface and not only on the details. On the contrary, the prominent coloring manages to produce clear and smooth boundaries. It combines the advantages of the mean and maximal coloring - a smooth image with clear boundaries. For example, these differences can be observed on the eye and the crown in Figure16. The prominent coloring better emphasizes the relevant details. This can also be seen in Figure 17. The prominent coloring is smoother than the maximal coloring and crisper than the mean coloring.

Moreover, the maximal and the mean coloring can generate spurious details or remove the true ones. For instance, observe the small cavities denoted by the yellow and magenta circles in Figure 18. The mean coloring completely removes them. The maximal coloring removes one cavity and changes the shape of the other.

\section{Conclusions}

This paper addressed the problem of automatic processing of scanned artifacts. The processing is based on a definition of a new field - the prominent field, which is a smooth direction field perpendicular to the feature curves. The prominent field is computed in interactive time (a couple of seconds for a 100,000-vertex model ). We demonstrated how to employ the prominent field for two applications: surface enhancement and artificial surface coloring, which emphasizes the object features. In both cases, the methods were applied to archaeological artifacts, which are typically noisy and suffered erosion over time. We showed that our results outperformed the results obtained by previous methods.

The applications received positive feedback from archaeologists from the Computerized Archaeological Laboratory at the Hebrew university of Jerusalem ${ }^{1}$. Their impression is that "it is not just a nice way to visualize and publish archaeological objects, but also an important research tool that improves the interpretation of the items especially with written material." Moreover, they intend to use this enhancement method in their future publications.

In the future, we intend to apply our prominent field to other applications, such as shape matching and reconstruction.

The data used in the project is available at http://webee.technion.ac.il/labs/cgm/ Computer-Graphics-Multimedia/Resources/Resources.html

Acknowledgements This research was supported in part by the Israel Science Foundation (ISF) 628/08, Olendorff foundation, the joint Technion University of Haifa research foundation, and the Goldbers fund for electronics research. We thank Dr. A. Gilboa and Zinman Institute of Archaeology for supplying us the models which were excavated at Tel Dor and for fruitful discussions on the topic.

\footnotetext{
1 http://archaeology.huji.ac.il/depart/computerized.asp
} 


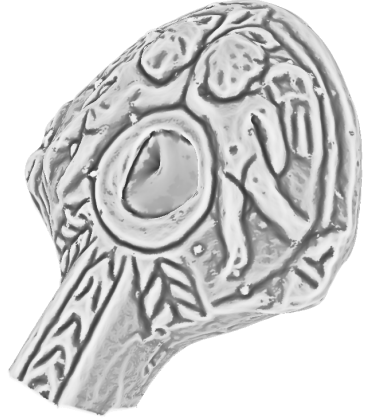

(a) Maximal-curvature coloring

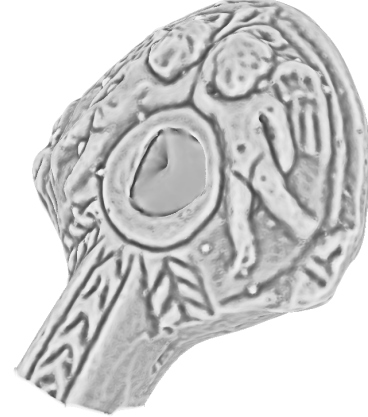

(b) Mean-curvature coloring

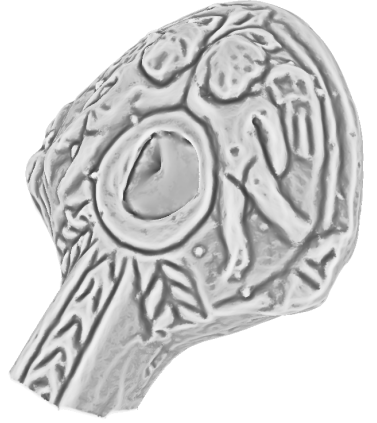

(c) Prominent coloring

Fig. 17 Comparison of various coloring methods. The prominent coloring combines the advantages of the mean and maximal coloring - a smooth image with clear boundaries.

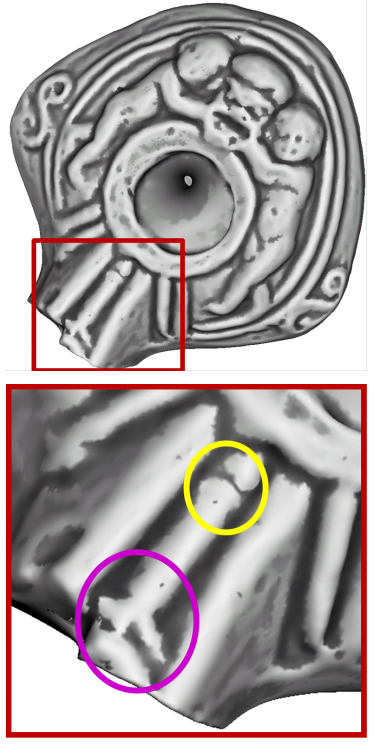

(a) Maximal-curvature coloring
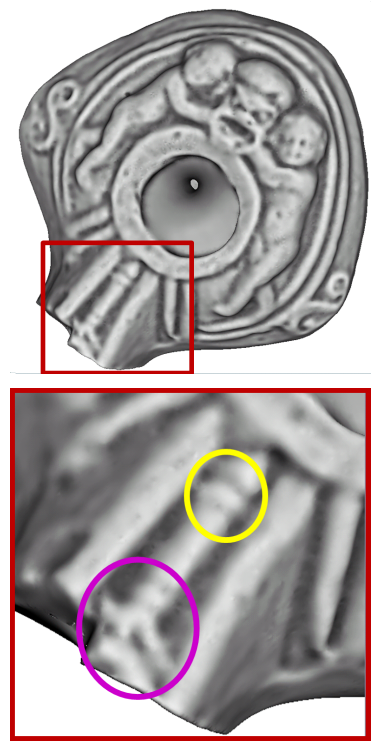

(b) Mean-curvature coloring
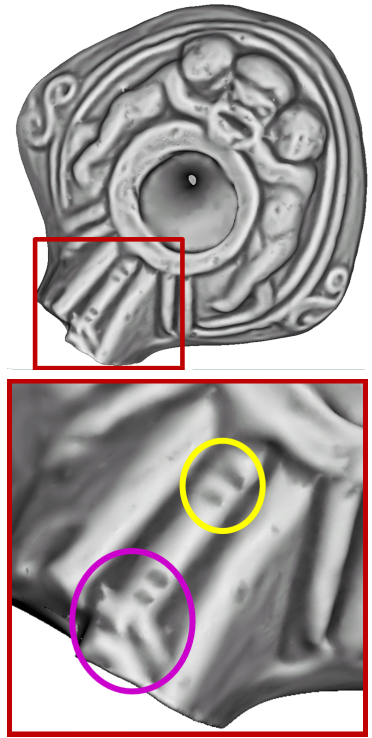

(c) Prominent coloring

Fig. 18 Comparison of various coloring methods on a Hellenistic lamp. With maximal curvature (a), the area inside the yellow circle appears as if it is divided into two parts and inside the magenta circle appears broken. With mean curvature (b) both area are blurred. The prominent coloring (c) better depicts these areas.

\section{References}

1. Bajaj, C., Xu, G.: Anisotropic diffusion of surfaces and functions on surfaces. ACM Transactions on Graphics (TOG) 22(1), 4-32 (2003)

2. Brown, B., Toler-Franklin, C., Nehab, D., Burns, M., Dobkin, D., Vlachopoulos, A., Doumas, C., Rusinkiewicz, S., Weyrich, T.: A system for high-volume acquisition and matching of fresco fragments: reassembling Theran wall paintings. ACM Trans. Graph. 27(3) (2008)

3. Cohen, J., Dunkan, D., Snyder, D., Cooper, J., Kumar, J., Hahn, S., Chen, D., Purnomo, B., Graettinger, J.: iclay: Digitizing cuneiform. In: Symposium on Virtual Reality, Archaeology and Cultural Heritage, pp. 135-143 (2004) 
4. Desbrun, M., Meyer, M., Schröder, P., Barr, A.: Implicit fairing of irregular meshes using diffusion and curvature flow pp. 317-324 (1999)

5. Do Carmo, M.P.: Differential geometry of curves and surfaces. Prentice-Hall (1976)

6. Eigensatz, M., Sumner, R., Pauly, M.: Curvature-domain shape processing. Comp. Graph. Forum 27(2), 241-250 (2008)

7. Fisher, M., Schroder, P., Desbrun, M., Hoppe, H.: Design of tangent vector fields. ACM Transactions on Graphics (TOG) 26(3), 56 (2007)

8. Fleishman, S., Drori, I., Cohen-Or, D.: Bilateral mesh denoising. ACM Trans. on Graph. 22(3), 950-953 (2003)

9. von Funck, W., Theisel, H., Seidel, H.: Vector field based shape deformations. ACM Transactions on Graphics (TOG) 25(3), 11-25 (2006)

10. Gooch, B., Sloan, P.J., Gooch, A., Shirley, P., Riesenfeld, R.F.: Interactive technical illustration. In: Symp. on Inter. 3D Graph., pp. 31-38 (1999)

11. Hertzmann, A., Zorin, D.: Illustrating smooth surfaces. Proceedings of the 27 th annual conference on Computer graphics and interactive techniques pp. 517-526 (2000)

12. Kindlmann, G., Whitaker, R., Tasdizen, T., Moller, T.: Curvature-based transfer functions for direct volume rendering: Methods and applications. In: IEEE Transactions on Visualization and Computer Graphics, pp. 67-76 (2003)

13. Koller, D., Trimble, J., Najbjerg, T., Gelfand, N., Levoy, M.: Fragments of the city: Stanford's digital forma urbis romae project. J. of Roman Arch. pp. 237-252 (2006)

14. Kolomenkin, M., Shimshoni, I., Tal, A.: Demarcating curves for shape illustration. ACM Trans. on Graph., SIGGRAPH Asia 27(4) (2008)

15. Kolomenkin, M., Shimshoni, I., Tal, A.: On edge detection on surfaces. CVPR pp. 2767-2774 (2009)

16. Kolomenkin, M., Shimshoni, I., Tal, A.: Prominent field for shape processing of archaeological artifacts. IEEE Workshop on eHeritage and Digital Art Preservation (ICCV) (2009)

17. Meyer, M., Desbrun, M., Schroder, P., Barr, A.H.: Discrete differential-geometry operators for triangulated 2-manifolds. In: VisMath, vol. B, pp. 187-217 (2002)

18. Ohtake, Y., Belyaev, A., Seidel, H.: Mesh smoothing by adaptive and anisotropic gaussian filter applied to mesh normals. Vis., Model., and Visual. pp. 203-210 (2002)

19. Ray, N., Li, W., Lévy, B., Sheffer, A., Alliez, P.: Periodic global parameterization. ACM Transactions on Graphics (TOG) 25(4), 148-195 (2006)

20. Rushmeier, H.: Eternal Egypt: experiences and research directions. Recording, Modeling and Visualization of Cultural Heritage pp. 22-27 (2006)

21. Rusinkiewicz, S., Burns, M., DeCarlo, D.: Exaggerated shading for depicting shape and detail. ACM Transactions on Graphics 25(3), 1199-1205 (2006)

22. Stam, J.: Flows on surfaces of arbitrary topology. ACM Transactions On Graphics (TOG) 22(3), 724-731 (2003)

23. Stern, E.: Excavations at dor. Tech. rep., Institute of Archaeology of the Hebrew University (1995)

24. Sun, X., Rosin, P., Martin, R., Langbein, F.: Fast and effective feature-preserving mesh denoising. IEEE Trans. on Vis. and Comp. Graph. 13(5), 925-938 (2007)

25. Tasdizen, T., Whitaker, R., Burchard, P., Osher, S.: Geometric surface smoothing via anisotropic diffusion of normals. IEEE Visualization, 2002 pp. 125-132 (2002)

26. Taubin, G.: A signal processing approach to fair surface design. Comp. grap. and interact. techn. pp. 351-358 (1995)

27. Toler-Franklin, C., Finkelstein, A., Rusinkiewicz, S.: Illustration of complex real-world objects using images with normals. In: NPAR-07, pp. 111-119 (2007)

28. Turk, G.: Texture synthesis on surfaces. ACM Transactions on Graphics (TOG) 19(3), 347-354 (2001)

29. Vrubel, A., Bellon, O., Silva, L.: A 3D Reconstruction Pipeline for Digital Preservation. CVPR pp. 2687 -2694 (2009)

30. Wei, L., Levoy, M.: Texture synthesis over arbitrary manifold surfaces. ACM Transactions on Graphics (TOG) 19(3), 355-360 (2001)

31. Yoshizawa, S., Belyaev, A., Seidel, H.: Smoothing by example: Mesh denoising by averaging with similarity-based weights. IEEE Int. Conf. on Shape Model. and App. pp. 9-19 (2006)

32. Zhang, E., Mischaikow, K., Turk, G.: Vector field design on surfaces. ACM Transactions on Graphics (TOG) 25(4), 1294-1326 (2006) 